\title{
An unexpected twist
}

This is an
exciting
situation
where we
know that we
can contribute
something
new and
unexpected
to the
fundamental
understanding
of magnetic
droplets

Magnetic droplets are nanoscale magnetic excitations with cores of magnetization that is reversed relative to the surrounding material. Theoretically predicted in the 1970s and experimentally confirmed in 2013, magnetic droplets have so far eluded direct imaging. Now, Johan Åkerman and collaborators, writing in Physical Review Letters, present scanning transmission X-ray microscopy images of magnetic droplets. The images reveal that the droplets are twice as large as expected, and suggest that previous theories were incomplete.

Creating stable droplets in environments where they can be imaged is challenging. Past experiments used orthogonal spin valves, which are structures that have a 'free layer' with magnetization normal to the plane and a 'fixed layer' with magnetization that is in-plane when the applied magnetic field is zero. However, once a magnetic field is applied to allow a droplet to nucleate in the free layer, the fixed layer magnetization tilts out of plane. This tilting breaks symmetry and subjects the droplet to a drift instability that changes its properties.
Åkerman and colleagues have devised an approach that keeps symmetry unbroken, and thus allows imaging of droplets in their original states. The approach involves changing the composition of the spin valve so that the fixed and free layers have strong perpendicular magnetic anisotropy and remain aligned. More specifically, the composition of the fixed layer is multilayers of Co-Pd and the free layer is composed of Co-Ni multilayers. Åkerman and colleagues report the nucleation of the magnetic droplets by observing changes in the electrical resistance of the droplets at sufficiently high magnetic field and current amplitudes.

Following this proof-of-principle, the droplets were imaged and shown to be perfectly circular, as expected. However, theory predicted that droplets should have the same diameter as the nanocontact. "Instead, the measurements revealed that the droplet diameter is about twice the diameter of the nanocontact," says Åkerman. "Clearly something was amiss with the theoretical predictions, and we immediately thought about possible mechanisms behind this.'

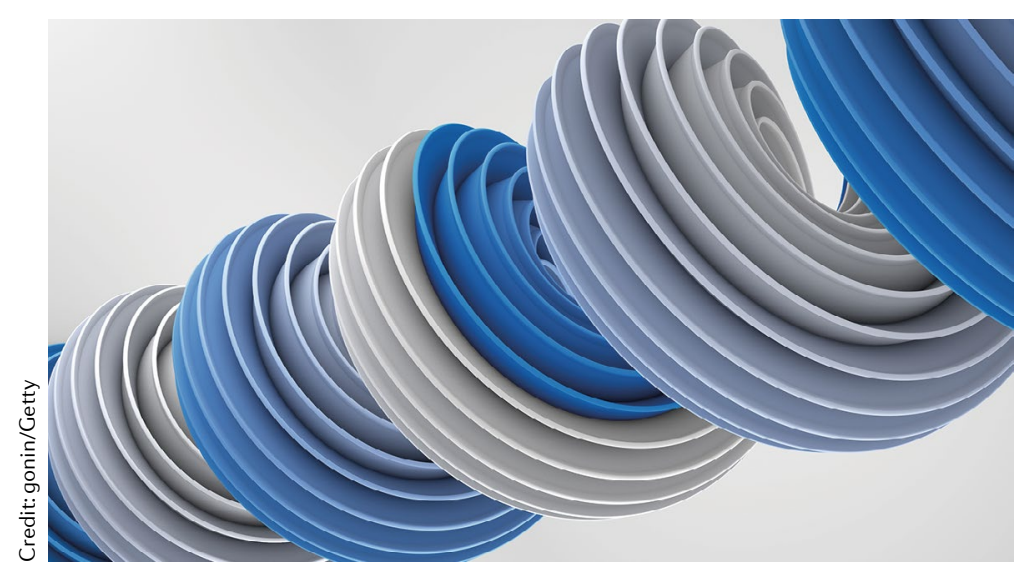

The key to the mystery was an earlier observation that currents from the nanocontacts tend to spread laterally in the spin valve. The perimeter of a magnetic droplet is a precessing magnetic domain wall, and like all magnetic domain walls it can be moved by in-plane electrical currents. Micromagnetic simulations reveal that the droplet perimeter is pushed outward by Zhang-Li torque, which acts when electrons interact with spatially and time-varying magnetization. Including this torque in simulations led to droplets that agreed with experiments in terms of size as well as shape.

In future studies, the researchers aim to focus on phenomena such as droplet merging, interactions between droplets in the free and fixed layers, and droplet-to-skyrmion transitions that may involve changes in the topology of the magnetization. "This is an exciting situation where we know that we can contribute something new and unexpected to the fundamental understanding of magnetic droplets," says Åkerman. There are also possible technological applications that involve harnessing the precession of the droplet magnetization for generating microwave signals. "We are now trying to create droplets in magnetic tunnel junctions. If we do this, then we might have found the best possible route towards generating high-power microwave signals in spin torque oscillators," concludes Åkerman.

Zoe Budrikis, Associate Editor, Nature Reviews Physics

ORIGINAL ARTICLE Chung, S. et al. Direct observation of Zhang-Li torque expansion of magnetic droplet solitons. Phys. Rev. Lett. 120, 217204 (2018) 